\title{
GENERALIZED GAUSSIAN ESTIMATES AND RIESZ MEANS OF SCHRÖDINGER GROUPS
}

\author{
SÖNKE BLUNCK
}

(Received 27 September 2004; revised 23 September 2005)

\author{
Communicated by A. H. Dooley
}

\begin{abstract}
We show that generalized Gaussian estimates for selfadjoint semigroups $\left(e^{-t A}\right)_{t \in \mathbb{R}_{+}}$on $L_{2}$ imply $L_{p^{-}}$ boundedness of Riesz means and other regularizations of the Schrödinger group $\left(e^{i t A}\right)_{t \in \mathbb{R}}$. This generalizes results restricted to semigroups with a heat kernel, which are due to Sjöstrand, Alexopoulos and more recently Carron, Coulhon and Ouhabaz. This generalization is crucial for elliptic operators $A$ that are of higher order or have singular lower order terms since, in general, their semigroups fail to have a heat kernel.
\end{abstract}

2000 Mathematics subject classification: primary 43A15; secondary 47A60.

\section{Introduction}

It is well known that the Schrödinger group $\left(e^{i t \Delta}\right)_{t \in \mathbb{R}}$ acts on $L_{p}\left(\mathbb{R}^{D}\right)$ only if $p=2[18]$. Various authors showed that suitable regularizations of the Schrödinger group such as the Riesz means

$$
t^{-\alpha} \int_{0}^{t}(t-s)^{\alpha-1} e^{i s \Delta} d s
$$

act even on $L_{p}\left(\mathbb{R}^{D}\right)$ for $p \neq 2$; see, for example, the works of Lanconelli [20] on boundedness of $(I-\Delta)^{-\alpha} e^{i t \Delta}$ and of Sjöstrand [26] on Riesz means. These results were extended by Alexopoulos [1] to Laplacians $\Delta$ on Lie groups and Riemannian manifolds where the heat semigroup satisfies Gaussian estimates.

Carron, Coulhon and Ouhabaz [10] generalized this approach to arbitrary selfadjoint operators $A$ on measured metric spaces $(\Omega, \mu, d)$ of some dimension $D$, that

(c) 2007 Australian Mathematical Society $1446-7887 / 07 \$ A 2.00+0.00$ 
is, $|B(x, \lambda r)| \leq C \lambda^{D}|B(x, r)|$ for all $x \in \Omega, r>0, \lambda \geq 1$, where $B(x, r)$ denotes the ball around $x$ of radius $r$ and $|B(x, r)|$ its volume. They showed $L_{p}$-boundedness of suitable regularizations of the Schrödinger group $\left(e^{i t A}\right)_{t \in \mathbb{R}}$ provided $A$ satisfies Gaussian estimates (GEs), that is, the $e^{-t A}$ have integral kernels $k_{t}(x, y)$ satisfying

$$
\left|k_{t}(x, y)\right| \leq\left|B\left(x, r_{t}\right)\right|^{-1} g\left(\frac{d(x, y)}{r_{t}}\right) \text { for all } x, y \in \Omega, t>0 .
$$

Here the $r_{t}$ are suitable positive radii and $g: \mathbb{R}_{+} \rightarrow \mathbb{R}_{+}$is a suitable decay function. The central part of [10] was to deduce from the GE (1) the following $L_{p} \rightarrow L_{p}$-norm estimate for the semigroup:

$$
\left\|e^{-z A}\right\|_{p \rightarrow p} \leq C_{\varepsilon}\left(\frac{|z|}{\operatorname{Re} z}\right)^{D|1 / 2-1 / p|+\varepsilon} \quad \text { for all } p \in[1, \infty], z \in \mathbb{C}_{+},
$$

where $\mathbb{C}_{+}:=\{z \in \mathbb{C} ; \operatorname{Re} z>0\}$. Then one can apply directly the following result on Riesz means and regularized groups due to El-Mennaoui [23] and Boyadzhiev and de Laubenfels [9]. Recall that if $X$ is a Banach space and $S \in \mathfrak{L}(X)$ is injective, then a strongly continuous family $(W(t))_{t \in \mathbb{R}}$ in $\mathfrak{L}(X)$ is called an $S$-regularized group if $W(0)=S$ and $W(s) W(t)=S W(s+t)$ for all $s, t \in \mathbb{R}$. Its generator $B$ is defined by $B=S^{-1} W^{\prime}(0)$ with maximal domain; see, for example, [13] for details.

Proposition A. Let $(\Omega, \mu)$ be a measure space, A a non-negative selfadjoint operator on $L_{2}(\Omega), p \in[1, \infty]$, and $\alpha>\beta \geq 0$ such that $\left\|e^{-2 A}\right\|_{p \rightarrow p} \leq C(|z| / \operatorname{Re} z)^{\beta}$ for all $z \in \mathbb{C}_{+}$.

(a) The following Riesz means $\left(I_{\alpha}(t)\right)_{t \in \mathbb{R}}$ are uniformly bounded on $L_{p}(\Omega)$ :

$$
I_{\alpha}(t):= \begin{cases}t^{-\alpha} \int_{0}^{t}(t-s)^{\alpha-1} e^{-i s A} d s & t \geq 0 \\ I_{\alpha}(-t)^{*} & t<0 .\end{cases}
$$

(b) If $\left(e^{-t A}\right)$ is bounded analytic of angle $\pi / 2$ on $L_{p}(\Omega)$, then $W_{\alpha}(t):=(I+A)^{-\alpha} e^{i t A}$ defines $a(I+A)^{-\alpha}$-regularized group on $L_{p}(\Omega)$ with generator $i$ A satisfying

$$
\left\|W_{\alpha}(t)\right\|_{p \rightarrow p} \leq C(1+|t|)^{\alpha} \quad \text { for all } t \in \mathbb{R} .
$$

Unfortunately, there are many important operators $A$ which do not satisfy GEs (in particular, [10] cannot be applied!). This occurs, for example, for elliptic operators $A$ that are of higher order or have singular lower order terms [12, 21]. However, in many of these cases $A$ still satisfies so-called generalized Gaussian estimates (GGEs); see $[11,25]$. By this we mean an estimate of the following type:

$$
\left\|\chi_{B\left(x, r_{t}\right)} e^{-t A} \chi_{B\left(y, r_{t}\right)}\right\|_{p_{o} \rightarrow p_{o}^{\prime}} \leq\left|B\left(x, r_{t}\right)\right|^{1 / p_{o}^{\prime}-1 / p_{o}} g\left(\frac{d(x, y)}{r_{t}}\right)
$$


for all $x, y \in \Omega, t>0$, and for some $p_{o} \in[1,2)$. The GGE (3) for $p_{o}=1$ is equivalent to the GE (1) [5, Proposition 2.9]. The central part of this paper is to deduce from the GGE (3) the following generalization and slight improvement of the $L_{p} \rightarrow L_{p}$-norm estimate (2) (Theorem 1.1 below), which improves a result of Davies [11]:

$$
\left\|e^{-z A}\right\|_{p \rightarrow p} \leq C\left(\frac{|z|}{\operatorname{Re} z}\right)^{D|1 / 2-1 / p|} \quad \text { for all } p \in\left[p_{o}, p_{o}^{\prime}\right], z \in \mathbb{C}_{+} .
$$

We obtain new results on regularizations of the Schrödinger group $\left(e^{i t A}\right)_{t \in \mathbb{R}}$, as before directly from Proposition $\mathrm{A}$, and for the optimal range $p \in\left[p_{o}, p_{o}^{\prime}\right], \alpha>D|1 / 2-1 / p|$; see Theorem 1.3 below. We want to mention that, for the class of operators $A$ satisfying the GGE (3), the interval $\left[p_{o}, p_{o}^{\prime}\right]$ is, in general, optimal for the existence of the semigroup $\left(e^{-t A}\right)_{t \in \mathbb{R}_{+}}$on $L_{p}$ [12], and the $\left\|e^{-z A}\right\|_{p \rightarrow p}$-estimate (4) is optimal also [3].

A singular integral theory based on GGEs allows us to extend other $L_{2}$-properties of $A$ (above the boundedness of regularizations of $\left(e^{i t A}\right)_{t \in \mathbb{R}}$ considered in this paper) to $L_{p}$ for $p \in\left(p_{o}, p_{o}^{\prime}\right)$. We mention the properties of having maximal regularity [5], an $H^{\infty}$ functional calculus [6] or Riesz transforms [7, 17]. In [4], this approach was applied to so-called 'spectral multipliers', which yields results of the type $F(A) \in \mathfrak{L}\left(L_{p}\right), p \in$ $\left(p_{o}, p_{o}^{\prime}\right)$ for more general functions $F$ than the $F_{\alpha, t}(x):=t^{-\alpha} \int_{0}^{t}(t-s)^{\alpha-1} e^{-i s x} d s$ corresponding to our Riesz means (that is, $F_{\alpha, t}(A)=I_{\alpha}(t)$ ). The advantage of the method in the present paper is that it allows us to include the cases $p=p_{o}, p_{o}^{\prime}$ and gives a direct approach for Schrödinger groups avoiding singular integral theory.

\section{Main results}

We begin with some basic notation and assumptions. For the rest of this paper, $(\Omega, \mu, d)$ is a metric measure space. By $B(x, r)$ we denote balls in $\Omega$ and by $|B(x, r)|$ or $v_{r}(x)$ their volume. For integral operators $T$, we denote their integral kernel by $k_{T}(x, y)$, that is, $T f(x)=\int k_{T}(x, y) f(y) d \mu(y)$.

1.1. Optimal estimates for $\left\|e^{-z A}\right\|_{p \rightarrow p}$ In order to deduce from Proposition $A$ optimal $L_{p}$-boundedness results for regularizations of Schrödinger groups $\left(e^{i t A}\right)_{t \in \mathbb{R}}$, one needs optimal $\left\|e^{-z A}\right\|_{p \rightarrow p}$-estimates. For the case of operators satisfying GEs, (almost) optimal $\left\|e^{-z A}\right\|_{p \rightarrow p}$-estimates are obtained in [10, Theorem 4.3] by making tricky use of the identity

$$
\left\|k_{T}(\cdot, y)\right\|_{2}^{2}=k_{T^{*} T}(y, y) .
$$

Here we optimize and generalize this method to GGEs. The right substitute of the above kernel identity can be seen in the elementary norm identity

$$
\|T\|_{p \rightarrow 2}^{2}=\left\|T^{*} T\right\|_{p \rightarrow p^{\prime}}
$$


This allows us to prove the following $\left\|e^{-z A}\right\|_{p \rightarrow p}$-estimate. The proof of this estimate will be given at the end of this subsection.

THEOREM 1.1. Let $(\Omega, \mu, d)$ be a space of dimension $D$ and $p \in[1,2)$. Let $A$ be a non-negative selfadjoint operator on $L_{2}(\Omega)$ such that

$$
\left\|\chi_{B\left(x, r_{t}\right)} e^{-t A} \chi_{B\left(y, r_{t}\right)}\right\|_{p \rightarrow p^{\prime}} \leq\left|B\left(x, r_{t}\right)\right|^{1 / p^{\prime}-1 / p} g\left(\frac{d(x, y)}{r_{t}}\right)
$$

for all $x, y \in \Omega, t \in \mathbb{R}_{+}$, where $r_{t}=t^{1 / m}$ and $g(s)=C \exp \left(-b s^{m /(m-1)}\right)$ for some $m \geq 2$, and $C, b>0$. Then $\left(e^{-t A}\right)_{t \in \mathbb{R}_{+}}$is bounded analytic of angle $\pi / 2$ on $L_{p}(\Omega)$, and we have

$$
\left\|e^{-z A}\right\|_{p \rightarrow p} \leq C\left(\frac{|z|}{\operatorname{Re} z}\right)^{D(1 / p-1 / 2)} \quad \text { for all } z \in \mathbb{C}_{+} .
$$

REMARK 1.2. (a) The Laplacian $A=-\Delta$ on $\Omega=\mathbb{R}^{D}$ shows the optimality of our result [3].

(b) Duality and interpolation with $\left\|e^{-z A}\right\|_{2 \rightarrow 2} \leq 1$ yield that $\left(e^{-t A}\right)_{t \in \mathbb{R}_{+}}$is bounded analytic of angle $\pi / 2$ on $L_{q}(\Omega)$ for all $q \in\left[p, p^{\prime}\right], q \neq \infty$, and we have

$$
\left\|e^{-z A}\right\|_{q \rightarrow q} \leq C\left(\frac{|z|}{\operatorname{Re} z}\right)^{D|1 / 2-1 / q|} \quad \text { for all } q \in\left[p, p^{\prime}\right], z \in \mathbb{C}_{+} .
$$

(c) In [11], Davies verified the hypothesis of Theorem 1.1 for elliptic operators $A$ of order $m \in 2 \mathbb{N}$ on $\Omega=\mathbb{R}^{D}$ and for $p:=(2 D /(D+m)) \vee 1$, but he only obtained the following weaker conclusion, see [11, Theorems 20 and 25]:

$$
\left\|e^{-z A}\right\|_{p \rightarrow p} \leq C\left(\frac{|z|}{\operatorname{Re} z}\right)^{2 D(1 / p-1 / 2)} \quad \text { for all } z \in \mathbb{C}_{+} .
$$

(d) For the special case $p=1$, our Theorem 1.1 is a slight improvement of [10, Theorem 4.3], where the following estimate is obtained for all $\varepsilon>0$ :

$$
\left\|e^{-z A}\right\|_{1 \rightarrow 1} \leq C_{\varepsilon}\left(\frac{|z|}{\operatorname{Re} z}\right)^{D / 2+\varepsilon} \quad \text { for all } z \in \mathbb{C}_{+} .
$$

Deducing from the $L_{p} \rightarrow L_{p^{\prime}}$ GGE in the hypothesis, an $L_{p} \rightarrow L_{2}$ GGE, and extending the latter to complex times are the main steps in the following short proof of Theorem 1.1.

PROOF OF THEOREM 1.1. We identify $g$ and $\widetilde{g}$, where $\widetilde{g}(s)=\widetilde{C} \exp \left(-\widetilde{b} s^{m /(m-1)}\right)$. By Proposition 3.1 (i) below, the $L_{p} \rightarrow L_{p^{\prime}}$ GGE in the hypothesis implies the following $L_{p} \rightarrow L_{2} \mathrm{GGE:}$

$$
\left\|\chi_{B\left(x, r_{t}\right)} e^{-t A} \chi_{B\left(y, r_{t}\right)}\right\|_{p \rightarrow 2} \leq\left|B\left(x, r_{t}\right)\right|^{1 / 2-1 / p} g\left(\frac{d(x, y)}{r_{t}}\right) \text { for all } t \in \mathbb{R}_{+} .
$$


By Theorem 2.1 below, the latter extends to complex times $z \in \mathbb{C}_{+}$as follows:

$$
\left\|\chi_{B\left(x, r_{z}\right)} e^{-z A} \chi_{B\left(y, r_{z}\right)}\right\|_{p \rightarrow 2} \leq\left|B\left(x, r_{z}\right)\right|^{1 / 2-1 / p}\left(\frac{|z|}{\operatorname{Re} z}\right)^{D(1 / p-1 / 2)} g\left(\frac{d(x, y)}{r_{z}}\right)
$$

for all $z \in \mathbb{C}_{+}$, where $r_{z}=(\operatorname{Re} z)^{1 / m-1}|z|$. This implies by Proposition 3.1 (ii) below for $R=(|z| / \operatorname{Re} z)^{-D(1 / p-1 / 2)} e^{-z A}$ :

$$
\left\|e^{-z A}\right\|_{p \rightarrow p} \leq C_{0}\left(\frac{|z|}{\operatorname{Re} z}\right)^{D(1 / p-1 / 2)} \quad \text { for all } z \in \mathbb{C}_{+} .
$$

It remains to show that $\left(e^{-z A}\right)_{z \in \mathbb{C}_{+}}$is strongly continuous in $L_{p}$ on all strict subsectors of $\mathbb{C}_{+}$. Arguing as in [24], but on $L_{p}$ instead of $L_{1}$, one obtains the strong continuity on subsectors from the previous $L_{p}-L_{p}$-estimate.

1.2. $L_{p}$-boundedness of regularizations of $\left(e^{i t A}\right)_{t \in \mathbb{R}} \quad$ Theorem 1.1 allows us to apply our approach given in the introduction, that is, to verify the $L_{p} \rightarrow L_{p}$ norm estimate

$$
\left\|e^{-z A}\right\|_{p \rightarrow p} \leq C\left(\frac{|z|}{\operatorname{Re} z}\right)^{D|1 / 2-1 / p|} \text { for all } p \in\left[p_{o}, p_{o}^{\prime}\right], z \in \mathbb{C}_{+}
$$

and to obtain $L_{p}$-boundedness of Riesz means and $(I+A)^{-\alpha}$-regularizations of $\left(e^{i t A}\right)_{t \in \mathbb{R}}$ directly from Proposition A. This yields the following result.

THEOREM 1.3. Let $(\Omega, \mu, d)$ be a space of dimension $D$ and $p_{o} \in[1,2)$. Let $A$ be a non-negative selfadjoint operator on $L_{2}(\Omega)$ such that

$$
\left\|\chi_{B\left(x, r_{t}\right)} e^{-t A} \chi_{B\left(y, r_{t}\right)}\right\|_{p_{o} \rightarrow p_{o}^{\prime}} \leq\left|B\left(x, r_{t}\right)\right|^{1 / p_{o}^{\prime}-1 / p_{o}} g\left(\frac{d(x, y)}{r_{t}}\right)
$$

for all $x, y \in \Omega, t \in \mathbb{R}_{+}$, where $r_{t}=t^{1 / m}$ and $g(s)=C \exp \left(-b s^{m /(m-1)}\right)$ for some $m \geq 2$, and $C, b>0$. Then we have for all $p \in\left[p_{o}, p_{o}^{\prime}\right]$ and $\alpha>D|1 / 2-1 / p|$ :

(a) The following Riesz means $\left(I_{\alpha}(t)\right)_{t \in \mathbb{R}}$ are uniformly bounded on $L_{p}(\Omega)$ :

$$
I_{\alpha}(t):= \begin{cases}t^{-\alpha} \int_{0}^{t}(t-s)^{\alpha-1} e^{i s A} d s & t \geq 0, \\ I_{\alpha}(-t)^{*} & t<0 .\end{cases}
$$

(b) If $p \neq \infty$ then $W_{\alpha}(t):=(I+A)^{-\alpha} e^{i t A}$ defines an $(I+A)^{-\alpha}$-regularized group $\left(W_{\alpha}(t)\right)_{t \in \mathbb{R}}$ on $L_{p}(\Omega)$, with generator $i A$ satisfying $\left\|W_{\alpha}(t)\right\|_{p \rightarrow p} \leq C(1+|t|)^{\alpha}$ for all $t \in \mathbb{R}$.

REMARK 1.4. (a) For the special case $p_{o}=1$ our Theorem 1.3 corresponds to Theorems 5.1-5.2 in [10]. 
(b) The Laplacian $A=-\Delta$ on $\Omega=\mathbb{R}^{D}$ shows the optimality of our result [3].

(c) In Remark 1.2 (c) above we already mentioned Davies' estimate [11]

$$
\left\|e^{-z A}\right\|_{p \rightarrow p} \leq C\left(\frac{|z|}{\operatorname{Re} z}\right)^{2 D|1 / 2-1 / p|} \quad \text { for all } p \in\left[p_{o}, p_{o}^{\prime}\right], z \in \mathbb{C}_{+}
$$

for elliptic operators $A$ of order $m \in 2 \mathbb{N}$ on $\Omega=\mathbb{R}^{D}$ and for $p_{o}=(2 D /(D+m)) \vee 1$. By Proposition A, this yields the conclusion of Theorem 1.3 for the range $\alpha>$ $2 D|1 / 2-1 / p|$. Our result yielding the optimal range $\alpha>D|1 / 2-1 / p|$ is new.

PROOF OF THEOREM 1.3. The assertions follow directly from Theorem 1.1 (in the form of Remark 1.2 (b)) and Proposition A for $\beta=D|1 / 2-1 / p|$.

1.3. Examples In this subsection, we give some examples of elliptic operators $A$ for which our Theorem 1.3 on regularizations of the Schrödinger group $\left(e^{i t A}\right)_{t \in \mathbb{R}}$ applies, that is, for which the following GGE holds:

$$
\left\|\chi_{B\left(x, r_{t}\right)} e^{-t A} \chi_{B\left(y, r_{t}\right)}\right\|_{p_{o} \rightarrow p_{o}^{\prime}} \leq\left|B\left(x, r_{t}\right)\right|^{1 / p_{o}^{\prime}-1 / p_{o}} C \exp \left(-b\left(\frac{d(x, y)}{r_{t}}\right)^{m /(m-1)}\right)
$$

for all $x, y \in \Omega, t \in \mathbb{R}_{+}, r_{t}=t^{1 / m}$ and for some $m \geq 2, p_{o} \in[1,2)$.

1.3.1. Higher order operators with bounded coefficients on $\mathbb{R}^{D} \quad$ These operators $A$ are given by forms $\mathfrak{a}: H^{k}\left(\mathbb{R}^{D}\right) \times H^{k}\left(\mathbb{R}^{D}\right) \rightarrow \mathbb{C}$ of the type

$$
\mathfrak{a}(u, v)=\int_{\mathbb{R}^{D}} \sum_{|\alpha|=|\beta|=k} a_{\alpha \beta} \partial^{\alpha} u \overline{\partial^{\beta} v} d x,
$$

where we assume $a_{\alpha, \beta}=\overline{a_{\beta, \alpha}} \in L_{\infty}\left(\mathbb{R}^{D}\right)$ for all $\alpha, \beta$ and Garding's inequality

$$
\mathfrak{a}(u, u) \geq \delta\left\|\nabla^{k} u\right\|_{2}^{2} \text { for all } u \in H^{k}\left(\mathbb{R}^{D}\right),
$$

for some $\delta>0$ and $\left\|\nabla^{k} u\right\|_{2}^{2}:=\sum_{|\alpha|=k}\left\|\partial^{\alpha} u\right\|_{2}^{2}$. Then $\mathfrak{a}$ is a closed symmetric form The associated operator $A$ is given by $u \in D(A)$, and $A u=g$ if and only if $u \in H^{k}$ and $\int g \bar{v} d x=\mathfrak{a}(u, v)$ for all $v \in H^{k}$.

In this situation, the GGE (6) holds for $p_{o}:=(2 D /(m+D)) \vee 1$ and $m:=2 k$; see, for example, [11] and [2, Section 1.7]. Hence the conclusion of Theorem 1.3 holds for all $p \in\left[p_{o}, p_{o}^{\prime}\right]$ and $\alpha>D|1 / 2-1 / p|$.

1.3.2. Schrödinger operators with singular potentials on $\mathbb{R}^{D}$ Now we study Schrödinger operators $A=-\Delta+V$ on $\mathbb{R}^{D}, D \geq 3$, where $V=V_{+}-V_{-}, V_{ \pm} \geq 0$ 
are locally integrable, and $V_{+}$is bounded for simplicity (for the general case, see for example [25]). We assume the following form bound:

$$
\int\left(V_{-} u\right) \bar{u} d x \leq \gamma\left(\|\nabla u\|_{2}^{2}+\left\langle V_{+} u, u\right\rangle\right)+c(\gamma)\|u\|_{2}^{2} \text { for all } u \in H^{1}\left(\mathbb{R}^{D}\right)
$$

and some $\gamma \in(0,1)$. Then the form sum $A:=-\Delta+V=\left(-\Delta+V_{+}\right)-V_{-}$is defined and the associated form is closed and symmetric with form domain $H^{1}\left(\mathbb{R}^{D}\right)$. By standard arguments using ellipticity and the Sobolev inequality, the GGE (6) holds for $p_{o}=2 D /(D+2)$ and $m=2$ (after replacing $A$ by $A+c(\gamma)$ ). Due to [21], $\left(e^{-t A}\right)_{t \in \mathbb{R}_{+}}$is bounded on $L_{q}\left(\mathbb{R}^{D}\right)$ for all $q \in\left(p_{\gamma}, p_{\gamma}^{\prime}\right)$ and

$$
p_{\gamma}:=\frac{2 D}{D(1+\sqrt{1-\gamma})+2(1-\sqrt{1-\gamma})}<\frac{2 D}{D+2} .
$$

Hence, by interpolation, one obtains the GGE (6) even for all $p_{o} \in\left(p_{\gamma}, 2\right)$. Thus, the conclusion of Theorem 1.3 holds for all $p \in\left(p_{\gamma}, p_{\gamma}^{\prime}\right)$ and $\alpha>D|1 / 2-1 / p|$.

1.3.3. Elliptic operators on Riemannian manifolds Let $A=-\Delta$ be the Laplacian on a Riemannian manifold $\Omega$. Let $d$ be the geodesic distance and $\mu$ the Riemannian measure. Assume that $\Omega$ satisfies the so-called volume doubling property and that the heat kernel $k_{t}(x, y)$ satisfies

$$
k_{t}(x, x) \leq C|B(x, \sqrt{t})|^{-1} \text { for all } x \in \Omega, t>0 .
$$

Then $\left(e^{t \Delta}\right)_{t \in \mathbb{R}_{+}}$satisfies GEs [16] or, equivalently, the GGE (6) for $p_{o}=1$ and $m=2$. Hence the results we obtain on regularizations of $\left(e^{-i t \Delta}\right)_{t \in \mathbb{R}}$ are contained in [10].

\section{Extension of GGEs for $\left(e^{-t A}\right)_{t \in \mathbb{R}_{+}}$to $\left(e^{-z A}\right)_{z \in \mathbb{C}_{+}}$}

Theorem 1.3 on regularizations of Schrödinger groups is based on GGEs of the type

$$
\left\|\chi_{B\left(x, r_{i}\right)} e^{-t A} \chi_{B\left(y, r_{t}\right)}\right\|_{p \rightarrow q} \leq\left|B\left(x, r_{t}\right)\right|^{1 / q-1 / p} g\left(\frac{d(x, y)}{r_{t}}\right) .
$$

Here we show, for analytic semigroups, how the latter estimate for real times $t$ extends to an estimate for complex times $z$ of the type

$$
\left\|\chi_{B\left(x, r_{z}\right)} e^{-z A} \chi_{B\left(y, r_{z}\right)}\right\|_{p \rightarrow q} \leq\left|B\left(x, r_{z}\right)\right|^{1 / q-1 / p} C(z) g\left(\frac{d(x, y)}{r_{z}}\right) .
$$

This is important for the proof of our $\left\|e^{-z A}\right\|_{p \rightarrow p}$-estimate in Theorem 1.1. Moreover, many other applications of GGEs require such complex time estimates, for example, the $H^{\infty}$ functional calculus; see, for example, [6, 14]. 
In our setting, we have to extend (7) to the whole right halfplane $\mathbb{C}_{+}$. This is crucial in order to obtain optimal results on Riesz means or more general so-called 'spectral multipliers' of selfadjoint semigroups; see $[4,10,15]$.

For extensions of (7) to strict subsectors of the sector of analyticity of the semigroup, one can usually choose $r_{z}:=r_{|z|}$ and $C(z)=1$ in $(8)$; see $[19,21]$. The latter choice is impossible on the whole of $\mathbb{C}_{+}$, as can be seen by fixing $x \neq y$ and by letting $|\arg z| \rightarrow \pi / 2$.

The right candidate for the constant $C(z)$ in $(8)$ is $C(z)=R(z)^{D(1 / p-1 / q)}$, where $R(z):=r_{z} / r_{\operatorname{Re} z}$. This is suggested by Proposition 3.1, Remark 3.2 below and confirmed by our following extension result. Its proof is given in Section 3 .

We consider the standard case $r_{t}=t^{1 / m}$ and $g(s)=C e^{-b s^{\omega}}$, which corresponds (for $\omega=m /(m-1)$ ) to the typical estimates for elliptic operators $A$ of order $m$; see $[2,11]$.

THEOREM 2.1. Let $(\Omega, \mu, d)$ be a space of dimension $D$ and $1 \leq p \leq p_{o} \leq q \leq \infty$. Let $\left(e^{-t A}\right)_{t \in \mathbb{R}_{+}}$be a semigroup of linear operators on $L_{p_{o}}(\Omega)$, which has a bounded and analytic extension to $\mathbb{C}_{+}$and satisfies, for all $t \in \mathbb{R}_{+}$,

$$
\left\|\chi_{B\left(x, r_{t}\right)} e^{-t A} \chi_{B\left(y, r_{t}\right)}\right\|_{p \rightarrow q} \leq\left|B\left(x, r_{t}\right)\right|^{1 / q-1 / p} C e^{-b\left(d(x, y) / r_{t}\right)^{\omega}},
$$

where $r_{t}=t^{1 / m}$ for some $m>0$ and $\omega \in(0,2 m)$. Then, for all $z \in \mathbb{C}_{+}$,

$$
\left\|\chi_{B\left(x, r_{z}\right)} e^{-z A} \chi_{B\left(y, r_{z}\right)}\right\|_{p \rightarrow q} \leq\left|B\left(x, r_{z}\right)\right|^{1 / q-1 / p} R(z)^{D(1 / p-1 / q)} C^{\prime} e^{-b^{\prime}\left(d(x, y) / r_{z}\right)^{\omega}},
$$

where $r_{z}:=\cos (\arg z)^{-1 / \omega}|z|^{1 / m}$ and $R(z):=r_{z} / r_{\operatorname{Re} z}=\cos (\arg z)^{-(1 / \omega+1 / m)}$.

REMARK 2.2. (a) For the classical case $\omega=m /(m-1), m \geq 2,(p, q)=(1, \infty)$ of GEs, our Theorem 2.1 corresponds to [10, Proposition 4.1].

(b) In [11], Davies verified the hypothesis of Theorem 2.1 for elliptic operators $A$ of order $m \in 2 \mathbb{N}$ on $\Omega=\mathbb{R}^{D}$ and for $p:=(2 D /(D+m)) \vee 1, q:=p^{\prime}, \omega=m /(m-1)$. He obtained precisely the conclusion of our Theorem 2.1; see [11, line (11) and Lemma 24].

A direct application of Theorem 2.1 is the following.

Corollary 2.3. Let $(\Omega, \mu, d)$ be a space of some dimension $D$ and $p \in[1,2)$. Let $A$ be a non-negative selfadjoint operator on $L_{2}(\Omega)$ such that

$$
\left\|\chi_{B\left(x, r_{t}\right)} e^{-t A} \chi_{B\left(y, r_{t}\right)}\right\|_{p \rightarrow p^{\prime}} \leq\left|B\left(x, r_{t}\right)\right|^{1 / p^{\prime}-1 / p} C \exp \left(-b\left(\frac{d(x, y)}{r_{t}}\right)^{\omega}\right),
$$

where $r_{t}=t^{1 / m}$ for some $m>1$ and $\omega \in(1, m]$. Then $A$ has a bounded $H^{\infty}$ functional calculus on $L_{q}(\Omega)$ for all $q \in\left(p, p^{\prime}\right)$. 
Recall that $A$ has a bounded $H^{\infty}$ functional calculus if we have an estimate

$$
\|f(A)\| \leq C\|f\|_{H^{\infty}\left(\Sigma_{\delta}\right)} \text { for all } f \in H^{\infty}\left(\Sigma_{\delta}\right)
$$

and some $\delta \in(0, \pi / 2)$. Here $H^{\infty}\left(\Sigma_{\delta}\right)$ denotes the space of all bounded holomorphic functions on the sector $\Sigma_{\delta}:=\{z ;|\arg (z)|<\delta\}$. This calculus was introduced by McIntosh; its construction and applications can be found in $[22,14,6]$.

PROOF OF COROLLARY 2.3. The conclusion of Theorem 2.1 holds for $q=p^{\prime}$ and, obviously, $A$ has a bounded $H^{\infty}$ functional calculus on $L_{2}(\Omega)$. Hence the assertion follows from [6, Theorem 1.2].

\section{Proof of Theorem 2.1}

We use the symbols $\preceq$ and $\succeq$ to indicate domination up to constants independent of the relevant parameters. Let $\sim$ indicate the validity of $\preceq$ and $\succeq$. While the GGEs considered in our main results enlighten the analogy to GEs, they are not convenient from a technical point of view. For this purpose, we use an equivalent type of GGE, which is provided by the following characterization [8, Proposition 2.1].

Proposition 3.1. Let $(\Omega, \mu, d)$ be a space of some dimension $D$ and $1 \leq p \leq$ $q \leq \infty$. Let $g(s):=C e^{-b s^{\omega}}$ for some $\omega>1, b, C>0$. Let $R$ be a linear operator and $r>0$.

(i) The following are equivalent:

(a) $\left\|\chi_{B(x, r)} R \chi_{B(y, r)}\right\|_{p \rightarrow q} \leq v_{r}(x)^{1 / q-1 / p} g(d(x, y) / r)$, for all $x, y \in \Omega$.

(b) $\left\|\chi_{B(x, r)} R \chi_{B(y, r)}\right\|_{p \rightarrow v} \leq v_{r}(x)^{1 / v-1 / p} g(d(x, y) / r)$, for all $x, y \in \Omega$ and $v \in[p, q]$.

(c) $\left\|\chi_{B_{1}} v_{r}^{\alpha} R v_{r}^{\beta} \chi_{B_{2}}\right\|_{p \rightarrow q} \leq g\left(d\left(B_{1}, B_{2}\right) / r\right)$, for all balls $B_{1}, B_{2} \subset \Omega$ and $\alpha, \beta \geq 0$ such that $\alpha+\beta=1 / p-1 / q$.

(ii) If (a) holds, then $\left\|v_{r}^{\alpha} R v_{r}^{\beta}\right\|_{u \rightarrow v} \leq C_{0}$, for all $p \leq u \leq v \leq q$ and all $\alpha, \beta \geq 0$ such that $\alpha+\beta=1 / u-1 / v$. Here $C_{0}$ is independent of $u, v, \alpha, \beta$ and $R, r$.

The statement (i) is written modulo identification of $g$ and $\widetilde{g}$, where $\widetilde{g}(s):=c g(s)^{a}$ for some constants $c, a>0$ independent of $R$ and $r$.

The preceding proposition and the following remark suggest that the right candidate for the constant $C(z)$ in complex time GGEs of the type (8) is $C(z)=R(z)^{D(1 / p-1 / q)}$, where $R(z):=r_{z} / r_{\mathrm{Re} z}$.

REMARK 3.2. Let $(\Omega, \mu, d)$ be a space of dimension $D>0$ and $p \in[1, \infty]$, $\alpha \geq 0$. Let $A$ be a non-negative selfadjoint operator on $L_{2}(\Omega)$ and $\left(r_{z}\right)_{z \in C_{+}}$a family 
in $\mathbb{R}_{+}$such that $r_{z} / r_{2 \operatorname{Re} z} \geq \varepsilon>0$ for all $z \in \mathbb{C}_{+}$. Suppose that $\left\|v_{r_{t}}^{\alpha} e^{-t A} v_{r_{t}}^{\alpha}\right\|_{p \rightarrow p^{\prime}} \leq C$ for all $t \in \mathbb{R}_{+}$. Then $\left\|v_{r_{z}}^{\alpha} e^{-2 z A} v_{r_{z}}^{\alpha}\right\|_{p \rightarrow p^{\prime}} \leq C^{\prime}\left(r_{z} / r_{2 \operatorname{Re} z}\right)^{2 D \alpha}$ for all $z \in \mathbb{C}_{+}$.

PROOF. Using the norm identity $\|T\|_{p \rightarrow 2}^{2}=\left\|T^{*} T\right\|_{p \rightarrow p^{\prime}}$ in the second step, the assertion is seen as follows:

$$
\begin{aligned}
\left\|v_{r_{z}}^{\alpha} e^{-2 z A} v_{r_{z}}^{\alpha}\right\|_{p \rightarrow p^{\prime}} & \leq\left\|v_{r_{z}}^{\alpha} e^{-z A}\right\|_{2 \rightarrow p^{\prime}}\left\|e^{-z A} v_{r_{z}}^{\alpha}\right\|_{p \rightarrow 2} \\
& =\left\|v_{r_{z}}^{\alpha} e^{-(2 \operatorname{Re} z) A} v_{r_{z}}^{\alpha}\right\|_{p \rightarrow p^{\prime}} \quad\left(e^{-z A}\right. \text { is normal, (5)) } \\
& \preceq\left(\frac{r_{z}}{r_{2 \operatorname{Re} z}}\right)^{2 D \alpha}\left\|v_{r_{2 \mathrm{Rez}}}^{\alpha} e^{(-2 \operatorname{Re} z) A} v_{r_{2 \mathrm{Re} z}}^{\alpha}\right\|_{p \rightarrow p^{\prime}} \quad(\operatorname{dim} \Omega=D) \\
& \preceq\left(\frac{r_{z}}{r_{2 \operatorname{Re} z}}\right)^{2 D \alpha} \quad \text { (by hypothesis). }
\end{aligned}
$$

The last preparation for the proof of Theorem 2.1 on the extension of GGEs to complex times is the following application of the three lines lemma. The proof is a straightforward modification of [11, Lemma 9].

LEMMA 3.3. Let $\omega, m \in \mathbb{R}_{+}$such that $0<\omega<2 m$ and $r_{z}:=\cos (\arg z)^{-1 / \omega}|z|^{1 / m}$ for all $z \in \mathbb{C}_{+}$. Let $\delta \geq 0$ and $C_{0}, b>0$. Let $X$ be a Banach space and $F: \mathbb{C}_{+} \rightarrow X$ an analytic function satisfying

$$
\begin{aligned}
& \|F(z)\| \leq C_{0} \cos (\arg z)^{-\delta} \quad \text { for all } z \in \mathbb{C}_{+}, \\
& \|F(t)\| \leq C_{0} \exp \left(-b r_{t}^{-\omega}\right) \text { for all } t \in \mathbb{R}_{+} .
\end{aligned}
$$

Then we have $\|F(z)\| \leq C_{0} 2^{\delta} \cos (\arg z)^{-\delta} e^{-(\omega / 2 m) b r} r_{2}^{-\omega}$ for all $z \in \mathbb{C}_{+}$.

ProOF. We define for all $\gamma \in(0, \pi / 2)$ :

$$
\begin{array}{ll}
g_{\gamma}^{ \pm}(z):=C_{0} \exp \left(-b \frac{e^{\mp i(\pi / 2-\omega \gamma / m)}}{\sin (\omega \gamma / m)} z^{-\omega / m}\right), & \pm \arg z \in[0, \gamma], \\
F_{\gamma}^{ \pm}(z):=F(z) g_{\gamma}^{ \pm}(z)^{-1}, & \pm \arg z \in[0, \gamma] .
\end{array}
$$

By hypothesis, we have for all $\gamma \in(0, \pi / 2)$ and $t>0$ :

$$
\begin{aligned}
\left\|F_{\gamma}^{ \pm}(t)\right\| & =\|F(t)\| C_{0}^{-1} \exp \left(b t^{-\omega / m}\right) \leq 1, \\
\left\|F_{\gamma}^{ \pm}\left(e^{ \pm i \gamma} t\right)\right\| & =\left\|F\left(e^{ \pm i \gamma} t\right)\right\| C_{0}^{-1} \leq \cos (\gamma)^{-\delta} .
\end{aligned}
$$

Hence the three lines lemma yields, for all $\gamma \in(0, \pi / 2)$ and $\theta \in[0,1]$,

$$
\left\|F_{\gamma}^{ \pm}\left(e^{ \pm i \gamma \theta} t\right)\right\| \leq 1^{1-\theta} \cos (\gamma)^{-\delta \theta} \leq \cos (\gamma)^{-\delta} .
$$


In other words, we have for all $\phi \in[0, \pi / 2), t>0$ and $\gamma \in[\phi, \pi / 2)$,

$$
\begin{aligned}
\left\|F\left(e^{ \pm i \phi} t\right)\right\| & =\left\|F_{\gamma}^{ \pm}\left(e^{ \pm i \phi} t\right)\right\|\left|g_{\gamma}^{ \pm}\left(e^{ \pm i \phi} t\right)\right| \\
& \leq \cos (\gamma)^{-\delta} C_{0} \exp \left(-b \sin \left(\frac{\omega}{m}(\gamma-\phi)\right) t^{-\omega / m}\right) .
\end{aligned}
$$

It remains to choose $\gamma=\pi / 4+\phi / 2$ and to deduce from the concavity of sin on $[0, \pi / 2]$ :

$$
\begin{aligned}
& \cos (\gamma)=\sin \left(\frac{\pi}{4}-\frac{\phi}{2}\right) \geq \frac{1}{2} \sin \left(\frac{\pi}{2}-\phi\right)=\frac{1}{2} \cos (\phi) \\
& \sin \left(\frac{\omega}{m}\left(\frac{\pi}{4}-\frac{\phi}{2}\right)\right) \geq \frac{\omega}{2 m} \sin \left(\frac{\pi}{2}-\phi\right)=\frac{\omega}{2 m} \cos (\phi)=\frac{\omega}{2 m} r_{e^{ \pm \dagger \phi} t}^{-\omega} t^{\omega / m} .
\end{aligned}
$$

PROOF OF THEOREM 2.1. We recall the basic volume estimate

$$
v_{s}(x) \leq C(1+s / r)^{D} v_{r}(x) \text { for all } x \in \Omega, r, s>0
$$

Now fix $\alpha:=1 / p_{o}-1 / q$ and $\beta:=1 / p-1 / p_{o}$. By Proposition 3.1 (ii), we obtain

$$
\left\|v_{r_{t}}^{\alpha} e^{-t A}\right\|_{p_{o} \rightarrow q},\left\|e^{-t A} v_{r_{t}}^{\beta}\right\|_{p \rightarrow p_{o}} \leq C \text { for all } t>0 .
$$

This allows for all $z \in \mathbb{C}_{+}, t \in(0, \operatorname{Re} z / 2)$ and $s>0$ the estimate

$$
\begin{aligned}
\left\|v_{s}^{\alpha} e^{-i A} v_{s}^{\beta}\right\|_{p \rightarrow q} & \leq\left\|v_{s}^{\alpha} e^{-t A}\right\|_{p_{o} \rightarrow q}\left\|e^{-(z-2 t) A}\right\|_{p_{o} \rightarrow p_{o}}\left\|e^{-t A} v_{s}^{\beta}\right\|_{p \rightarrow p_{o}} \\
& \leq\left(1+\frac{s}{r_{t}}\right)^{D \alpha}\left\|v_{r_{t}}^{\alpha} e^{-t A}\right\|_{p_{o} \rightarrow q}\left(1+\frac{s}{r_{t}}\right)^{D \beta}\left\|e^{-t A} v_{r_{t}}^{\beta}\right\|_{p \rightarrow p_{o}} \\
& \preceq\left(1+\frac{s}{r_{t}}\right)^{D(1 / p-1 / q)}, \quad \text { since } \alpha+\beta=1 / p-1 / q
\end{aligned}
$$

Denoting $z=e^{i \theta} r$ for $z \in \mathbb{C}_{+}$, we note that

$$
\begin{aligned}
1+\frac{s}{(\operatorname{Re} z / 2)^{1 / m}} & \leq(2 / \cos \theta)^{1 / m}\left(1+\frac{s}{r^{1 / m}}\right) \\
& \leq(2 / \cos \theta)^{1 / m} \sqrt{2}\left|1+\frac{s}{z^{1 / m}}\right| \\
& =C \cos (\arg z)^{-1 / m}\left|1+\frac{s}{z^{1 / m}}\right| \text { for all } z \in \mathbb{C}_{+}, s>0,
\end{aligned}
$$

which combines with the preceding estimate for $t \nearrow \operatorname{Re} z / 2$ to give

$$
\left\|v_{s}^{\alpha} e^{-z A} v_{s}^{\beta}\right\|_{p \rightarrow q} \preceq \cos (\arg z)^{-(D / m)(1 / p-1 / q)}\left|1+\frac{s}{z^{1 / m}}\right|^{D(1 / p-1 / q)} .
$$


Denote $g(u):=C e^{-b u^{\omega}}$ and fix balls $B_{1}, B_{2} \subset \Omega$. We have for all $s, t>0$,

$$
\begin{aligned}
\left\|\chi_{B_{1}} v_{s}^{\alpha} e^{-t A} v_{s}^{\beta} \chi_{B_{2}}\right\|_{p \rightarrow q} & \leq\left(1+\frac{s}{r_{t}}\right)^{D(\alpha+\beta)}\left\|\chi_{B_{1}} v_{r_{t}}^{\alpha} e^{-t A} v_{r_{t}}^{\beta} \chi_{B_{2}}\right\|_{p \rightarrow q} \quad(\text { by }(9)) \\
& \leq\left(1+\frac{s}{r_{t}}\right)^{D(1 / p-1 / q)} g\left(d\left(B_{1}, B_{2}\right) r_{t}^{-1}\right)
\end{aligned}
$$

since $\alpha+\beta=1 / p-1 / q$. Here we used the hypothesis, and in the last step, Proposition 3.1 (i). For fixed $s>0$, we consider the analytic function $F: \mathbb{C}_{+} \rightarrow$ $\mathfrak{L}\left(L_{p}(\Omega), L_{q}(\Omega)\right)$ defined by

$$
F(z):=\left(1+\frac{s}{z^{1 / m}}\right)^{-D(1 / p-1 / q)} \chi_{B_{1}} v_{s}^{\alpha} e^{-z A} v_{s}^{\beta} \chi_{B_{2}} .
$$

We have shown the following two bounds:

$$
\begin{aligned}
& \|F(t)\|_{p \rightarrow q} \preceq g\left(d\left(B_{1}, B_{2}\right) t^{-1 / m}\right) \quad \text { for all } t>0 \text {, } \\
& \|F(z)\|_{p \rightarrow q} \preceq \cos (\arg z)^{-(D / m)(1 / p-1 / q)} \text { for all } z \in \mathbb{C}_{+} \text {. }
\end{aligned}
$$

We apply Lemma 3.3 (to $g\left(d\left(B_{1}, B_{2}\right) \cdot\right.$ ) instead of $g$ ) and obtain

$$
\|F(z)\|_{p \rightarrow q} \preceq \cos (\arg z)^{-\frac{D}{m}(1 / p-1 / q)} g\left(d\left(B_{1}, B_{2}\right) r_{z}^{-1}\right) \text { for all } z \in \mathbb{C}_{+},
$$

where $r_{z}=\cos (\arg z)^{-1 / \omega}|z|^{1 / m}$. This means for all $z \in \mathbb{C}_{+}$and $s>0$,

$$
\begin{aligned}
& \left\|\chi_{B_{1}} v_{s}^{\alpha} e^{-z A} v_{s}^{\beta} \chi_{B_{2}}\right\|_{p \rightarrow q} \\
& \quad \preceq\left|1+\frac{s}{z^{1 / m}}\right|^{D(1 / p-1 / q)} \cos (\arg z)^{-(D / m)(1 / p-1 / q)} g\left(d\left(B_{1}, B_{2}\right) r_{z}^{-1}\right) .
\end{aligned}
$$

Choosing $s=r_{z}$ yields

$$
\begin{aligned}
\left\|\chi_{B_{1}} v_{r_{z}}^{\alpha} e^{-z A} v_{r_{z}}^{\beta} \chi_{B_{2}}\right\|_{p \rightarrow q} \\
\quad \preceq\left|1+\frac{r_{z}}{z^{1 / m}}\right|^{D(1 / p-1 / q)} \cos (\arg z)^{-(D / m)(1 / p-1 / q)} g\left(d\left(B_{1}, B_{2}\right) r_{z}^{-1}\right) \\
\quad \preceq \cos (\arg z)^{-D(1 / \omega+1 / m)(1 / p-1 / q)} g\left(d\left(B_{1}, B_{2}\right) r_{z}^{-1}\right) .
\end{aligned}
$$

By Proposition 3.1 (i) and $\alpha+\beta=1 / p-1 / q$, the above estimate is equivalent to

$$
\begin{aligned}
& \left\|\chi_{B\left(x, r_{z}\right)} e^{-z A} \chi_{B\left(y, r_{z}\right)}\right\|_{p \rightarrow q} \\
& \quad \preceq v_{r_{z}}(x)^{1 / q-1 / p} \cos (\arg z)^{-D(1 / \omega+1 / m)(1 / p-1 / q)} g\left(\frac{d(x, y)}{r_{z}}\right) .
\end{aligned}
$$




\section{Acknowledgement}

I would like to thank Thierry Coulhon for many helpful comments on the first version of this paper.

\section{References}

[1] G. Alexopoulos, ' $L^{p}$ bounds for spectral multipliers from Gaussian estimates on the heat kernel', preprint.

[2] P. Auscher an $\mathrm{Ph}$. Tchamitchian, 'Square root problem for divergence operators and related topics', Astérisque 249 (1998).

[3] W. Arendt, O. El Mennaoui and M. Hieber, 'Boundary values of holomorphic semigroups', Proc. Amer. Math. Soc. 125 (1997), 635-647.

[4] S. Blunck, 'A Hörmander-type spectral multiplier theorem for operators without heat kernel', Ann. Scuola Norm. Sup. Pisa Cl. Sci. (5) 2 (2003), 449-459.

[5] S. Blunck and P. C. Kunstmann, 'Weighted norm estimates and maximal regularity', Adv. Differential Equations 7 (2002), 1513-1532.

[6] - 'Calderon-Zygmund theory for non-integral operators and the $H^{\infty}$ functional calculus', Rev. Mat. Iberoamericana 19 (2003), 919-942.

[7] —-, 'Weak type ( $p, p$ ) estimates for Riesz transforms', Math. Z. 247 (2004), 137-148.

[8] — - 'Generalized Gaussian estimates and the Legendre transform', J. Operator Theory 53 (2005), 351-365.

[9] K. Boyadzhiev and R. de Laubenfels, 'Boundary values of holomorphic semigroups', Proc. Amer. Math. Soc. 118 (1993), 113-118.

[10] G. Carron, T. Coulhon and E. M. Ouhabaz, 'Gaussian estimates and $L_{p}$-boundedness of Riesz means', J. Evol. Equ. 2 (2002), 299-317.

[11] E. B. Davies, 'Uniformly elliptic operators with measurable coefficients', J. Funct. Anal. 132 (1995), 141-169.

[12] - 'Limits on $L^{p}$-regularity of self-adjoint elliptic operators', J. Differential Equations 135 (1997), 83-102.

[13] R. de Laubenfels, 'Integrated semigroups, C-semigroups and the abstract Cauchy problem', Semigroup Forum 41 (1990), 83-95.

[14] X. T. Duong and A. McIntosh, 'Singular integral operators with non-smooth kernels on irregular domains', Rev. Mat. Iberoamericana 15 (1999), 233-265.

[15] X. T. Duong, A. Sikora and E. M. Ouhabaz, 'Plancherel type estimates and sharp spectral multipliers', J. Funct. Anal. 196 (2002), 443-485.

[16] A. Grigor'yan, 'Gaussian upper bounds for the heat kernel on an arbitrary manifolds', J. Differential Geom. 45 (1997), 33-52.

[17] S. Hofmann and J. M. Martell, ' $L^{p}$ bounds for Riesz transforms and square roots associated to second order elliptic operators', Publ. Mat. 47 (2003), 497-515.

[18] L. Hörmander, 'Estimates for translation invariant operators in $L^{p}$ spaces', Acta Math. 104 (1960), 93-140.

[19] P. C. Kunstmann, $L_{p}$-spectral properties of elliptic differential operators Habilitationsschrift, (Karlsruhe, 2002).

[20] E. Lanconelli, 'Valutazioni in $L^{p}\left(\mathbb{R}^{n}\right)$ della soluzione del problema di Cauchy per l'equazione di Schrödinger', Boll. Un. Mat. Ital. (4) 1 (1968), 591-607. 
[21] V. Liskevich, Z. Sobol and H. Vogt, 'On $L_{p}$-theory of $C_{0}$-semigroups associated with second order elliptic operators II', J. Funct. Anal. 193 (2002), 55-76.

[22] A. McIntosh, 'Operators which have an $H^{\infty}$ functional calculus', in: Miniconference on operator theory and partial differential equations (North Ryde), Proc. Center Math. Appl. Austral. Nat. Univ. 14 (Australian National Univ., Canberra, 1986) pp. 210-231.

[23] O. El Mennaoui, Trace des semi-groupes holomorphes singuliers à l'origine et comportement asymptotique (Ph.D. Thesis, University of Franche-Comté, France, 1992).

[24] E. M. Ouhabaz, 'Gaussian estimates and holomorphy of semigroups', Proc. Amer. Math. Soc. 123 (1995), 1465-1474.

[25] G. Schreieck and J. Voigt, 'Stability of the $L_{p}$-spectrum of generalized Schrödinger operators with form small negative part of the potential', in: Functional Analysis (eds. ?. Pietsch, ?. Ruess and H. Vogt), Proc. Essen 1991, Bierstedt (Marcel-Dekker, New York, 1994).

[26] S. Sjöstrand, 'On the Riesz means of the solutions of the Schrödinger equation', Ann. Scuola Norm. Sup. Pisa (3) 24 (1970), 331-348.

email: sonkeblunck@yahoo.fr 